\title{
Delays and Continuation of Hospital Visits Among HIV-Infected Persons and AIDS Cases in Japan
}

\author{
Shuji Hashimoto ', Takao Matsumoto ${ }^{2}$, Masaki Nagai ${ }^{3}$, Yutaka Matsuyama ${ }^{1}$, Yosikazu Nakamura ${ }^{4}$, \\ Tamami Umeda ${ }^{5}$, Mitsuhiro Kamakura ${ }^{6}$, Seiichi Ichikawa ${ }^{7}$, Satoshi Kimura ${ }^{8}$, Kazuo Fukutomi ${ }^{9}$, \\ and Masahiro Kihara ${ }^{10}$
}

This study attempts to clarify the distribution patterns of delay between HIV transmission and the first hospital visit among HIV-infected persons and AIDS cases in Japan except those infected through blood products. Such hospital visit patterns were analyzed, and the rates of reporting for HIV/AIDS surveillance among diagnosed HIV-infected persons and AIDS cases in hospitals were shown. From 1991 to 1997, a survey and subsequent follow-up were conducted among HIV-infected persons and AIDS cases diagnosed at 74 hospitals in Tokyo. The numbers of HIV-infected persons and AIDS cases were 590 and 208, respectively. The percentage of patients whose estimated date of HIV transmission was obtained ranged $23-41 \%$ among Japanese and non-Japanese HIV-infected persons and AIDS cases. Among these patients, $28 \%$ to $86 \%$ showed a 3 -year delay between HIV transmission and their first hospital visit. The rate of HIV-infected persons who continued to visit hospitals within 1 year after their first visit was $77 \%$ for Japanese and $45 \%$ for non-Japanese; among those after 1 year or more following their first hospital visit the rate was more than $80 \%$ among Japanese and over $70 \%$ among non-Japanese. The rate of reporting to HIV/AIDS surveillance among diagnosed HIV-infected persons and AIDS cases was $90 \%$ or more after 1994 in Japan. The delay between HIV transmission and the first hospital visit was suggested to be very long. Not a few patients stopped visiting hospitals after only a short time. Most diagnosed HIV-infected persons and AIDS cases were reported to the surveillance system of Japan. J Epidemiol, $2000 ; 10: 65-70$

HIV, AIDS, delay of hospital visits, continuation of hospital visits, surveillance

HIV/AIDS has a profound impact on the health of peoples worldwide. WHO reported that the cumulative number of AIDS cases was more than 1.8 million by the end of 1997 , and that the number of HIV-infected persons was estimated to be more than 30 million ${ }^{1,2}$. In Japan, according to the report on HIV/AIDS surveillance by the Ministry of Health and Welfare of Japan ${ }^{3,4)}$, the numbers of HIV-infected persons and AIDS cases, excluding those infected through blood coagulation factor products, were reported to be 2490 and 1056, respectively, by the end of 1997 .

Most HIV-infected persons have no specific symptoms for a long time after HIV transmission. The delay between HIV

Received March 10, 1999; accepted May 26, 1999.

${ }^{1}$ Department of Biostatistics/Epidemiology and Preventive Health Sciences, School of Health Sciences and Nursing, University of Tokyo.

${ }^{2}$ Department of General Medicine, Juntendo University School of Medicine.

${ }^{3}$ Department of Public Health, Saitama Medical School.

${ }^{4}$ Department of Health Science, Division of Epidemiology and Community Health, Jichi Medical School.

${ }^{5}$ Division of International Cooperation, National institute of Infectious Diseases.

${ }^{6}$ Department of Preventive Medicine and Public Health, Keio University School of Medicine.

${ }^{7}$ Laboratory of Public Health, Faculty of Hygienic Sciences, Kanagawa Prefectural College of Nursing and Medical Technology.

${ }^{8}$ Department of Infection Control and Prevention, School of Medicine, University of Tokyo.

${ }^{9}$ National Institute of Public Health.

${ }^{10}$ Department of Epidemiology, Kanagawa Cancer Center Research Institute.

Address for correspondence : Shuji Hashimoto, Department of Biostatistics/Epidemiology and Preventive Health Sciences, School of Health Sciences and Nursing, University of Tokyo, Hongo 7-3-1, Bunkyo-ku, Tokyo 113-0033 Japan. 
transmission and diagnosis of HIV infection may be very long ${ }^{2,5,6)}$. Reducing this delay is important for the treatment of HIV-infected persons and for preventing the further transmission of HIV ${ }^{7,8)}$. However, to study the delay between HIV transmission and diagnosis of HIV infection is very difficult because of uncertainty regarding the date of HIV transmission ${ }^{\text {? }}$. After the diagnosis of HIV infection, for HIV-infected persons and AIDS cases, continuation of hospital visits is necessary for their treatment. The continuation status of hospital visits among these patients has not been sufficiently investigated ${ }^{8,10)}$.

The most important information source for HIV and AIDS trends is HIV/AIDS surveillance. Among the most critical problems of HIV/AIDS surveillance is the low coverage rate ${ }^{3,11-13)}$. At the very least, the surveillance must have high reporting rates among the diagnosed HIV-infected persons and AIDS cases ${ }^{13)}$. The rate reported to AIDS surveillance in the US was $92 \%{ }^{14}$, and the reporting rate of diagnosed HIV-infection in one State was more than $75 \%{ }^{15}$. These rates have not been established in Japan yet ${ }^{16)}$.

This study attempts to clarify the distribution patterns of delay between HIV transmission and the first hospital visit among HIV-infected persons and AIDS cases in Japan except those infected through blood products. Such hospital visit patterns were analyzed, and the rates of reporting for HIV/AIDS surveillance among diagnosed HIV-infected persons and AIDS cases in hospitals were shown.

\section{MATERIALS AND METHODS}

The survey and subsequent follow-up for the indicated HIVinfected persons and AIDS cases diagnosed at 74 selected hospitals in Tokyo were conducted during 1991-1997. The patients diagnosed in 1991 and before were registered in the year 1991, and the patients diagnosed from 1992 to 1997 were registered annually. Information on the patients obtained by physicians from the registration included hospital name, patient number (coded by each hospital), the date and diagnostic status (HIV or AIDS) of the first visit to the hospital, refer- rals by other hospitals, nationality, sex, date of birth, source of exposure to HIV infection, estimated date of HIV infection based on patient recall, and reporting to the HIV/AIDS surveillance of the Ministry of Health and Welfare, Japan. In the subsequent follow-up, information on patients included current status (alive or dead), record of visiting the hospital and reasons for discontinuing visits (move to another hospital, departure from Japan or unknown reasons). Among patients who moved to other hospitals that were also included in this survey, their hospital visits were assumed to continue.

The number of patients registered was shown in Table 1. HIV-infected persons included 429 Japanese and 161 nonJapanese. AIDS cases included 147 Japanese and 61 nonJapanese. Transmission routes of HIV among Japanese and non-Japanese HIV-infected persons and AIDS cases were comprised of homosexual/bisexual contacts $31-60 \%$, heterosexual contacts $26-54 \%$, and other and unknown routes 10 $26 \%$.

For patients whose estimated date of HIV transmission was obtained, distribution patterns of the delay between HIV transmission and the first hospital visit were calculated. These distribution patterns were also calculated for patients not referred by other hospitals because such patients would have thus visited these other hospitals before making their first visit to ours. The continuation rates of visiting hospitals each year after the first hospital visit, and the reasons for discontinuing the visits were analyzed. The rates of reporting to HIV/AIDS surveillance were calculated. These analyses were conducted for Japanese and non-Japanese nationalities because there would be differences in medical care use between these 2 groups.

\section{RESULTS}

\section{Delay between HIV transmission and the first hospital visit}

The analysis of patients whose estimated date of HIV transmission was obtained was shown in Table 2. HIV-infected persons were $41 \%$ Japanese and $29 \%$ non-Japanese, while AIDS cases were $24 \%$ Japanese and $23 \%$ non-Japanese. There were

Table 1. Number of subjects.

\begin{tabular}{llrr}
\hline & Transmission routes of HIV & Japanese & Non-Japanese \\
\hline \multirow{2}{*}{$\begin{array}{llrl}\text { HIV-infected } \\
\text { persons }\end{array}$} & Homosexual/bisexual contacts & $211(49.2)$ & $50(31.1)$ \\
& Heterosexual contacts & $176(41.0)$ & $87(54.0)$ \\
& Other and unknown routes & $42(9.8)$ & $24(14.9)$ \\
\cline { 2 - 4 } & Total & $429(100)$ & $161(100)$ \\
\hline \multirow{2}{*}{ AIDS cases } & Homosexual/bisexual contacts & $88(59.9)$ & $19(31.1)$ \\
& Heterosexual contacts & $38(25.9)$ & $26(42.6)$ \\
& Other and unknown routes & $21(14.3)$ & $16(26.2)$ \\
\cline { 2 - 4 } & Total & $147(100)$ & $61(100)$ \\
\hline
\end{tabular}


small differences in the rates between patients with and without referral by other hospitals.

Distribution patterns of the delay between HIV transmission and the first hospital visit were shown in Figure 1. Among Japanese HIV-infected persons, such delay patterns were $36 \%$ in less than 1 year, $25 \%$ in $1-2$ years, $9 \%$ in $2-3$ years, and $28 \%$ in more than 3 years. Patients with delays of more than 3 years were $69 \%$ in Japanese AIDS cases, $37 \%$ in non-Japanese HIVinfected persons, and $86 \%$ in non-Japanese AIDS cases. There were small differences in the delay between patients with and without referral by other hospitals.

\section{Continuation of hospital visits}

The numbers of Japanese HIV-infected persons observed were 429 at the first hospital visit, 263 after 1 year, 187 after 2 years, and 125 after 3 years. The numbers of non-Japanese HIV-infected persons observed were 161 at the first visit, 56 after 1 year, 34 after 2 years, and 18 after 3 years.

The continuation status of visiting hospitals among HIVinfected persons each year after the first visit was shown in Figure 2. Among Japanese HIV-infected persons, 77\% continued to visit hospitals within 1 year after their first visit. Other developments included death $2 \%$, move to other hospitals $7 \%$, departure from Japan $1 \%$, and unknown reasons for discontinuing visits $14 \%$. After 1 year or more following the first hospital visit, more than $80 \%$ of the patients continued to visit the hospitals. The mortality rate of patients increased with the years after the first visit. Inversely, there was an annual decrease in the percentage of patients whose reasons for discontinuing the visits were unknown.

Among non-Japanese HIV-infected persons, $45 \%$ continued to visit the hospitals within 1 year after their first visit. Other developments included death $1 \%$, move to other hospitals $8 \%$, departure from Japan $23 \%$, and unknown reasons for discon- tinuing visits $24 \%$. After 1 year or more following the first hospital visit, more than $70 \%$ of the patients continued to visit the hospital. The percentage of patients who departed Japan after 1 year or more following the first visit was as high as among those who departed Japan within the first year. The percentage of the patients whose reasons for discontinuing the visits were unknown after 1 year or more following the first visit, was lower than among those who stopped within the first year.

\section{Reporting to HIV/AIDS surveillance}

Rates of reporting to HIV/AIDS surveillance among diagnosed HIV-infected persons and AIDS cases were shown in Table 3. The reporting rates among HIV-infected persons were $91 \%$ among Japanese and $84 \%$ among non-Japanese, and those for AIDS cases were $90 \%$ among Japanese and $84 \%$ among non-Japanese.

Rates of reporting to HIV/AIDS surveillance each year after the first hospital visit were shown in Figure 3. The reporting rates among both HIV-infected persons and AIDS cases increased for both Japanese and non-Japanese, reaching $90 \%$ or more after 1994.

\section{DISCUSSION}

Our study subjects were HIV-infected persons and AIDS cases diagnosed at 74 selected hospitals in Tokyo up to the end of 1997. Institutions surveyed included leading hospitais for the treatment of HIV infection and AIDS in Tokyo. The numbers of HIV-infected persons reported to the HIV/AIDS surveillance of Japan by the end of 1997 were 1300 Japanese and 1190 non-Japanese ${ }^{3)}$, while AIDS cases numbered 758 Japanese and 298 non-Japanese. Although the numbers of our subjects in Table 1 did not strictly coincide with these figures, we considered that our subjects included the majority of HIV-

Table 2. Proportions of patients whose estimated dates of HIV transmission were obtained.

\begin{tabular}{|c|c|c|c|c|c|}
\hline & & \multirow{2}{*}{ Nationality } & \multicolumn{2}{|c|}{ Date of HIV transmission } & \multirow{2}{*}{ Total } \\
\hline & & & obtained & not obtained & \\
\hline \multirow[t]{4}{*}{ All patients } & HIV-infected & Japanese & $176(41.0)$ & $253(59.0)$ & $429(100)$ \\
\hline & persons & Non-Japanese & $46(28.6)$ & $115(71.4)$ & $161(100)$ \\
\hline & AIDS cases & Japanese & $36(23.8)$ & $112(76.2)$ & $147(100)$ \\
\hline & & Non-Japanese & $14(23.0)$ & $47(77.0)$ & $61(100)$ \\
\hline \multirow{4}{*}{$\begin{array}{l}\text { Patients without } \\
\text { referral by } \\
\text { other hospitals }\end{array}$} & HIV-infected & Japanese & $118(49.0)$ & $123(51.0)$ & $241(100)$ \\
\hline & persons & Non-Japanese & $38(33.3)$ & $76(66.7)$ & $114(100)$ \\
\hline & AIDS cases & Japanese & $26(34.7)$ & $49(65.3)$ & $75(100)$ \\
\hline & & Non-Japanese & $11(25.6)$ & $32(74.4)$ & $43(100)$ \\
\hline
\end{tabular}



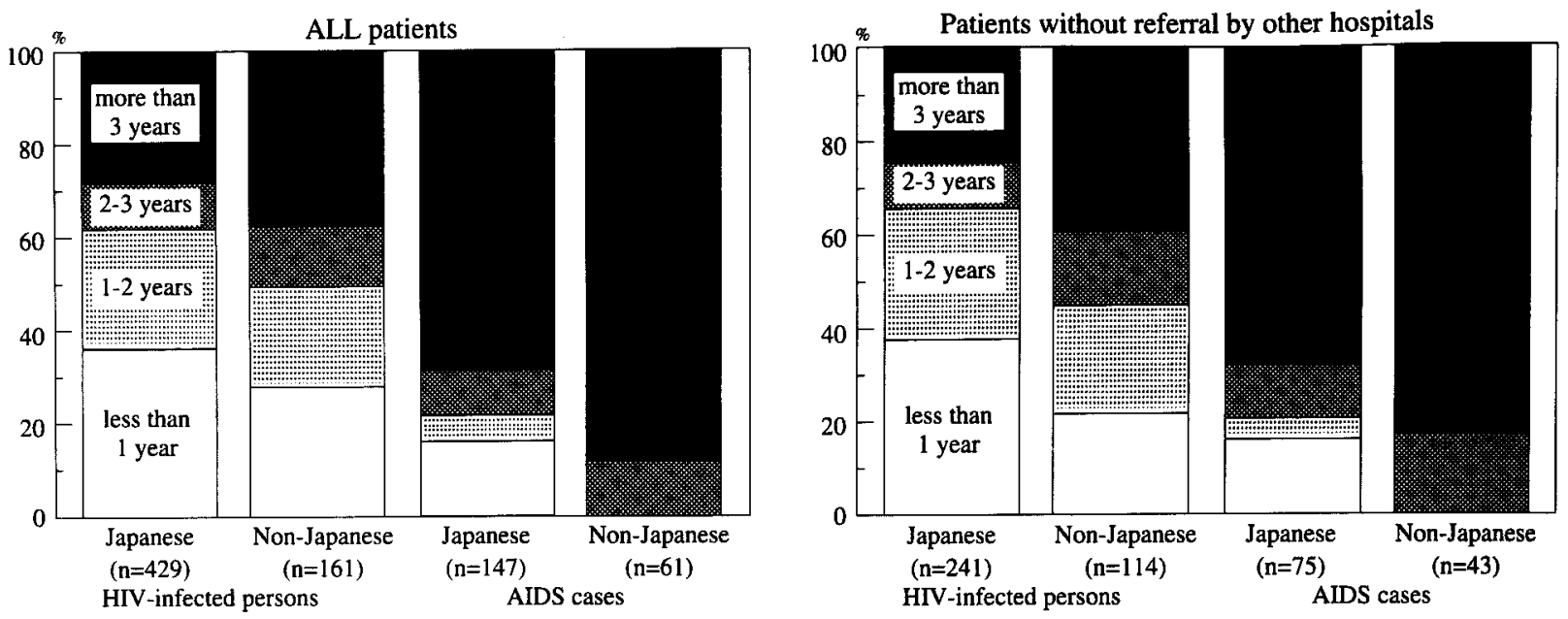

Figure 1. Distribution pattern of the delay between HIV transmission and the first hospital visit.

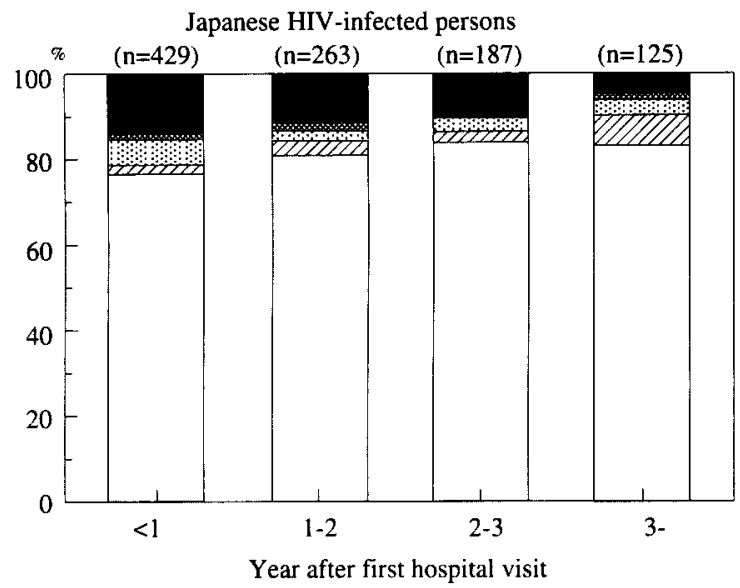

$\square$ Continued to visit $\square$ Dead

图 Departed Japan Unknown reasons

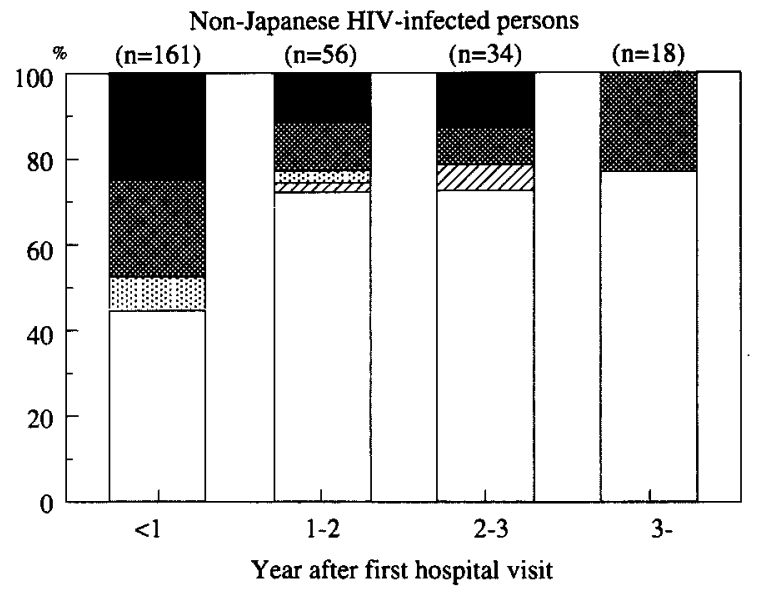

$\square$ Continued to visit $\square$ Dead

緼 Departed Japan Unknown reasons goved to other hospital

Figure 2. Continuation status of visiting hospitals among HIV-infected persons by the year after the first visit.

Table 3. Rates of reporting to HIV/AIDS surveillance.

\begin{tabular}{llcccc}
\hline & \multirow{2}{*}{ Nationality } & \multicolumn{3}{c}{ To HIV/AIDS surveillance } & \multirow{2}{*}{ Total } \\
\cline { 3 - 5 } & & reported & not reported & unknown & \\
\hline \multirow{2}{*}{$\begin{array}{l}\text { HIV-infected } \\
\text { persons }\end{array}$} & Japanese & $392(91.4)$ & $9(2.1)$ & $28(6.5)$ & $429(100)$ \\
& Non-Japanese & $135(83.9)$ & $7(4.3)$ & $19(11.8)$ & $161(100)$ \\
\hline AIDS cases & Japanese & $132(89.8)$ & $7(4.8)$ & $8(5.4)$ & $147(100)$ \\
& Non-Japanese & $51(83.6)$ & $5(8.2)$ & $5(8.2)$ & $61(100)$ \\
\hline
\end{tabular}



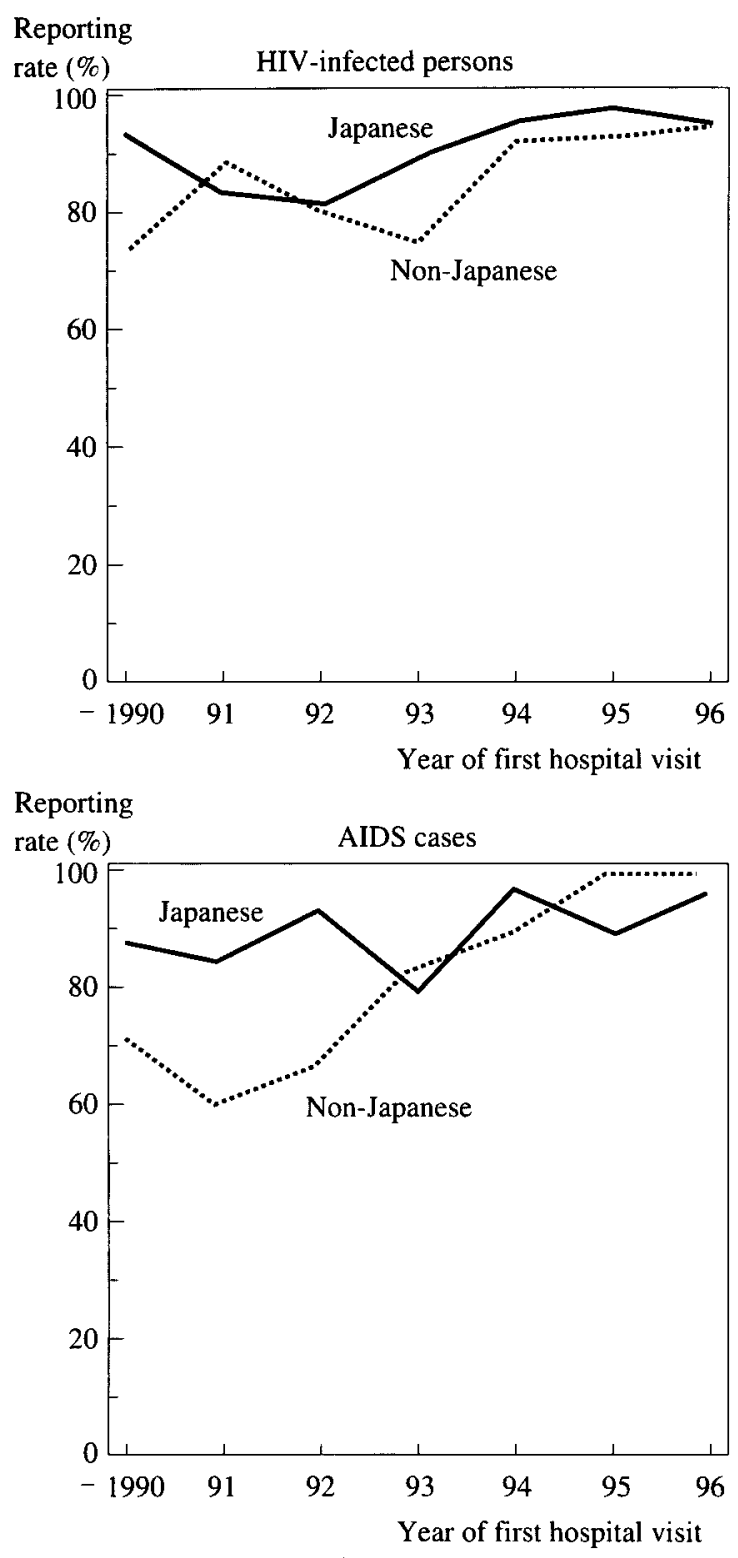

Figure 3. Rates of reporting to HIV/AIDS surveillance.

infected persons and AIDS cases diagnosed in Tokyo.

Patients who delayed more than 3 years between HIV transmission and their first hospital visit accounted for $28-86 \%$ (calculated from patients whose estimated dates of HIV transmission were obtained), and they were $24-41 \%$ of all patients. Our data supported the idea that the delay was so long that promoting voluntary HIV testing and counseling for HIV-infected persons had become more necessary ${ }^{2,5,6)}$.

HIV-infected persons who discontinued hospital visits except for reasons of death, movement to other hospitals or departure from Japan, were $14-24 \%$ within 1 year after the first visit, and decreased each year. A previous study reported that a follow-up loss among HIV-infected persons who presented themselves for care at a hospital in the US was $14 \%$ over 1.6 years ${ }^{10}$. The reason for this discrepancy in continuation of hospital visits is unknown, although patients who received no follow-up care were included in our study and were excluded in the above-mentioned study. Several studies reported that seeking medical care among HIV-infected persons and AIDS cases was associated with some factors including race, sex and drug use ${ }^{10,11}$. In our study, the proportion of patients who discontinued hospital visits among non-Japanese HIV-infected persons 
was higher than among Japanese cases. These findings suggested that not a few patients soon stopped visiting hospitals, and that it was important to investigate the reasons in detail.

The rate of reporting to the HIV/AIDS surveillance of Japan among diagnosed HIV-infected persons and AIDS cases reached more than $90 \%$ after 1994 . Although the reporting was not complete, we considered the rates high enough to demonstrate a trend in HIV infection and AIDS ${ }^{14,155}$. However, the coverage rates among all HIV-infected persons including cases not diagnosed would not be high because the delay between HIV transmission and the first hospital visit was very long $2,12,13$. It is important to estimate and improve the coverage rates.

A major limitation in our study is the uncertainty of information, including estimated dates of HIV transmission and reasons for stopping hospital visits. Information on the estimated date of HIV transmission was obtained through interviews by physicians. If HIV transmissions originate from experiences such as blood transfusion and travel abroad, HIV-infected persons and AIDS cases might recall their estimated date of HIV transmission. In Japan, HIV transmissions through blood transfusions have been few because $100 \%$ of blood bags were screened for HIV after $1986^{3,4)}$. HIV transmissions in foreign countries among Japanese HIV-infected persons and AIDS cases were reported to be $19 \%$ or more ${ }^{37}$. Although we failed to confirm the distribution patterns of delay between HIV transmission and the first hospital visit, it became clear that the delay was very long. Data on whether patients continued or discontinued their hospital visits were quite reliable, but the reasons for stopping the visits (e.g., departure from Japan or switching hospitals) were not so reliable because information was obtained from physician interviews. Although the distribution patterns of visiting hospitals may be biased, the suggestion that not a few patients soon discontinued their hospital visits may be valid. The information on reporting to HIV/AIDS surveillance was not confirmed by the surveillance data because the information did not include a patient's name and address, so individuals could not be identified ${ }^{3)}$. Although the rates of reporting to surveillance were not completely accurate, the general finding of high reporting rates would not be significantly affected.

\section{ACKNOWLEDGEMENTS}

This study was supported by a Grant-in-Aid from the Ministry of Health and Welfare, Japan for Research on HIV/AIDS.

\section{REFERENCES}

1. World Health Organization. Weekly epidemiological record. 1997;72:197-204.

2. World Health Organization. Report on the global HIV/AIDS epidemic - December 1997, 1997.

3. AIDS Surveillance Committee, Ministry of Health and Welfare of Japan ed. Annual surveillance report of HIV/AIDS in Japan, 1997, 1997.

4. Kihara M, Ichikawa S, Kihara M, Yamasaki S. Descriptive epidemiology of HIV/AIDS in Japan, 19851994. J AIDS 1997;14 (Suppl.2):S3-S12.

5. Wortley PM, Chu SY, Diaz T, et al. HIV testing patterns: where, why, and when were persons with AIDS tested for HIV? AIDS 1995;9:487-492.

6. Friedland GH. Early treatment for HIV: The time has come. N Engl J Med 1990;322:1000.

7. Francis DP, Anderson RE, Gorman ME, Fenstersheib M. Targeting AIDS prevention and treatment toward HIV-1infected persons: the concept of early intervention. JAMA 1989;262:2572-2576.

8. Toomey KE, Cates Jr W. Partner notification for the prevention of HIV infection, AIDS 1989;3(suppl 1):557-562.

9. Polk BF, Fox R, Brookmeyer R, et al. Predictors of the acquired immnodeficiency syndrome developing in a cohort of seropositive homosexual men. $N$ Eng $J$ Med 1987;316:61-66.

10. Chaisson RE, Keruly JC, Moore RD. Race, sex, drug use, and progression of human immunodeficiency virus. $N$ Eng J Med 1995;333:751-756.

11. Mor V, Fleishman JA, Dresser M, Piette J. Variation in health service use among HIV-infected patients. Med Care 1992;30:17-29.

12. Centers for Disease Control and Prevention. HIV/AIDS surveillance report. 1997.

13. Public Health Laboratory Service AIDS Center, Scottish Center for Infection and Environmental Health. AIDS/HIV quarterly surveillance tables: UK data to end September 1997.

14. Rosenblum L, et al. The completeness of AIDS case reporting, 1988: a multisite collaborative surveillance project. Am J Public Health 1992;82:1495-1499.

15. Meyer PA, Jones JJ, Garrison CZ. Completeness of reporting of diagnosed HIV-infected hospital patients. AIDS 1994;7:1067-1073.

16. Hashimoto S, Fukutomi K, Ichikawa S, Morio S, Shinmura K, Soda K. Study on trends of reported numbers of HIV-infected and AIDS cases based on the AIDS surveillance data until 1994. Jpn J Public Health 1992;42:1091-1098. 